\title{
NEUROIMMUNOLOGY
}

\section{IL-17: good fear no tears}

\author{
Rejane RUA, Nathalie PUJOL
}

Aix Marseille Univ, INSERM, CNRS, CIML, Centre d'Immunologie de Marseille-Luminy, Turing

Centre for Living Systems, Marseille, France

Corresponding authors rua@ciml.univ-mrs.fr \& pujol@ciml.univ-mrs.fr

\begin{abstract}
Cytokines are well-known mediators of the immune response but recently pleiotropic roles in the central nervous system have started to be uncovered. IL-17 directly modulates fear behaviour in mice.
\end{abstract}

IL17a is a canonical pro-inflammatory cytokine mainly produced by a group of $\mathrm{T}$ helper cells ( $T_{H} 17 \mathrm{CD}^{+} \mathrm{T}$ cells). It is also expressed by innate-like $\gamma \delta \mathrm{T}$ cells at barrier sites such as the gut mucosa, the skin, the lungs and the meninges, a tissue that envelops the central nervous system, essential for its protection. IL 17a is critical in the defence against microbial infections but can also promote systemic inflammation ${ }^{1}$. In neuroinflammatory diseases like multiple sclerosis, stroke, and Alzheimer's disease, it can also induce permeabilization of the bloodbrain barrier, recruitment of immune cells and excitotoxicity ${ }^{2}$. Under homeostatic conditions, the cellular source and the role in the brain of this cytokine was, however, unclear. In the current issue of Nature Immunology, Kipnis and colleagues reveal that the cytokine IL-17a affects neuronal functions independently of its proinflammatory activity, and dictates cognitive behaviours. This represents a further layer in neuro-immune interactions, reflecting the ancient role of cytokines in behavioural responses ${ }^{3,4}$ (Figure 1).

Kipnis and colleagues first demonstrate that homeostatic IL-17a is mainly produced by $ү \delta ~ T$ cells in the external leaflet of the meninges, the dura mater. Further exploring the ontology and specificity of meningeal $y \delta T$ cells in rodents, they demonstrate that fetal-derived $\gamma \delta T$ seed the meninges right after birth, proliferate massively during the first days of life and produce IL17a at steady state. Comparison with splenic $y \delta$ T cells indicate that meningeal $y \delta ~ T$ cells have a clear independent transcriptional signature. Postnatally, their activity seems to be governed at least in part by the microbiota.

Could secretion of this cytokine in the meninges affect brain function? Kipnis and colleagues investigate the homeostatic role of IL17a using behavioural tests. Wild-type mice usually avoid unprotected open areas in mazes and open-field tests. By using mice lacking $\gamma \delta T$ cells or by locally injecting a $y \delta T$ cell receptor (TCR)-blocking antibody, the authors show that in the absence of $\gamma \delta \mathrm{T}$ cell signalling, mice spend more time in unprotected open areas. This risky behaviour is also found in mice lacking IL-17a, but can be rescued by local IL-17a injection. The authors then show that the IL-17a receptor subunit a (IL-17Ra) is present in glutamatergic neurons of the prefrontal cortex (PFC), a brain region involved in regulating cognitive processes ${ }^{5}$. The transcriptomic mRNA signature of wild-type versus IL-17Ra-deficient PFC suggests that IL-17Ra ablation inhibits PFC activity. IL-17a release from meningeal $y \delta$ T cells thus increases the basal level of vigilance to modulate anxiety-behaviour. There are still open questions; the precise mode of action of IL-17a, for example, is unknown. Indeed, its concentration in the cerebrospinal fluid between the IL-17a-producing cells in the meninges and the target cells in the brain parenchyma is too low to be detected. 
This study supports the growing body of evidence that IL-17 cytokines fine-tune physiological and cognitive responses, beyond their pathogenic role in neuroinflammatory diseases. Recent work by Ribeiro et al. indicates that homeostatic IL-17a produced by meningeal $\gamma \delta$ T cells indirectly stimulates the hippocampal network through the production of neurotrophic factors by glial cells which promotes short-term memory ${ }^{6}$. This might indicate other pathways for IL$17 \mathrm{a}$ to act on cognition. But this finding is questioned by the current work by Kipnis and colleagues who could not reproduce memory impairment in IL-17a-deficient mice. Other studies have focused on the role of IL-17a in autism-like disorders. In a rodent model of social deficit, lipopolysaccharide (LPS) injection in the adult increases IL-17a production, which directly stimulates cortical network to restore social behaviour ${ }^{7}$. In contrast, embryonic IL-17a signalling results in social impairment in models of prenatal inflammation ${ }^{8}$. This highlights the complexity of IL-17 actions that depend on the context, the time of action, the site of production, and the concomitant production of other cytokines, such as IL-4, IL-6 and IFN- - , which are also involved in cognitive and social behaviours ${ }^{4}$. In addition, IL-17 cytokines affect the peripheral nervous system in mice. For instance, $y \delta$ T cells produce IL-17f, another member of the IL-17 family in response to cold. This promotes sympathetic innervation of the adipose tissue and maintains body temperature ${ }^{9}$. Moreover, IL-17a regulates the activation threshold of nociceptive neurons, and contributes to mechanical hyperalgesia ${ }^{10,11}$.

Importantly, the neuronal functions of IL-17 seem to be conserved in invertebrates (Figure 1). Wild-type $C$. elegans nematodes will concentrate at the border of a bacterial lawn, the thickest and more hypoxic part of this food source, avoiding high concentrations of oxygen. In a forward genetic screen for mutants that suppress this bordering phenotype, de Bono and colleagues identified an IL-17 cytokine and its receptors, as well as the downstream complex ACT-1-IRAKIKB. IL-17 signalling is required in one hub interneuron to increase its response to presynaptic input from oxygen sensors. This allows $C$. elegans to persistently escape high oxygen concentrations ${ }^{3}$. Thus, IL-17 signalling promotes aversion to noxious stimuli much like in the mouse (i.e. potentially hazardous open spaces). It is also required for associative learning. But surprisingly, neuronal IL-17 signalling in C. elegans suppresses intestinal defence mechanisms against infection and has a detrimental effect on longevity ${ }^{12}$.

Taken together, these observations highlight the importance of taking a broad view of the nonimmune functions of 'inflammatory cytokines' ${ }^{13}$ and their associated signalling pathways. IL17 neuronal activity allows integration of sensory inputs and appropriate cognitive behaviour across distantly-related species. Coordination of the immune response with an appropriate behaviour (escape, learning, social interactions) likely increases organisms' fitness. Notably, the sole TLR in C. elegans is also active in sensory neurons and is involved in the avoidance of pathogenic bacteria via sensing of carbon dioxide levels ${ }^{14,15}$.

These studies provide important avenues for future research. As exemplified by IL-17, the signalling pathways and molecular effects of 'immune' cytokines on neuronal and glial cells are still poorly understood. The dissection of such pathways in the nematode is one lead to follow ${ }^{12}$. In addition, in contrast to inflammatory conditions, the regulation of homeostatic cytokine production is still unclear. The potential implication of this work for humans needs further evaluation as it could help the design of pro-cognitive therapies.

\section{References}

1. McGeachy, M.J., Cua, D.J. \& Gaffen, S.L. The IL-17 Family of Cytokines in Health and Disease. Immunity 50, 892-906 (2019).

2. Cipollini, V., Anrather, J., Orzi, F. \& Iadecola, C. Th17 and Cognitive Impairment: Possible Mechanisms of Action. Front Neuroanat 13, 95 (2019). 
3. Chen, C. et al. IL-17 is a neuromodulator of Caenorhabditis elegans sensory responses. Nature 542, 43-48 (2017).

4. Filiano, A.J., Gadani, S.P. \& Kipnis, J. How and why do T cells and their derived cytokines affect the injured and healthy brain? Nat Rev Neurosci 18, 375-384 (2017).

5. Helfrich, R.F. \& Knight, R.T. Cognitive neurophysiology of the prefrontal cortex. Handb Clin Neurol 163, 35-59 (2019).

6. Ribeiro, M. et al. Meningeal gammadelta T cell-derived IL-17 controls synaptic plasticity and short-term memory. Sci Immunol 4 (2019).

7. Reed, M.D. et al. IL-17a promotes sociability in mouse models of neurodevelopmental disorders. Nature 577, 249-253 (2020).

8. Choi, G.B. et al. The maternal interleukin-17a pathway in mice promotes autism-like phenotypes in offspring. Science 351, 933-939 (2016).

9. Hu, B. et al. gammadelta T cells and adipocyte IL-17RC control fat innervation and thermogenesis. Nature 578, 610-614 (2020).

10. Ebbinghaus, M. et al. Interleukin-17A is involved in mechanical hyperalgesia but not in the severity of murine antigen-induced arthritis. Sci Rep 7, 10334 (2017).

11. Segond von Banchet, G. et al. Neuronal IL-17 receptor upregulates TRPV4 but not TRPV1 receptors in DRG neurons and mediates mechanical but not thermal hyperalgesia. Mol Cell Neurosci 52, 152-160 (2013).

12. Flynn, S.M. et al. MALT-1 mediates IL-17 neural signaling to regulate C. elegans behavior, immunity and longevity. Nat. Commun. 11, 2099 (2020).

13. Kipnis, J. Multifaceted interactions between adaptive immunity and the central nervous system. Science 353, 766-771 (2016).

14. Brandt, J.P. \& Ringstad, N. Toll-like Receptor Signaling Promotes Development and Function of Sensory Neurons Required for a C. elegans Pathogen-Avoidance Behavior. Curr Biol 25, 2228-2237 (2015).

15. Pradel, E. et al. Detection and avoidance of a natural product from the pathogenic bacterium Serratia marcescens by Caenorhabditis elegans. Proc Natl Acad Sci U S A 104, 2295-2300 (2007). 



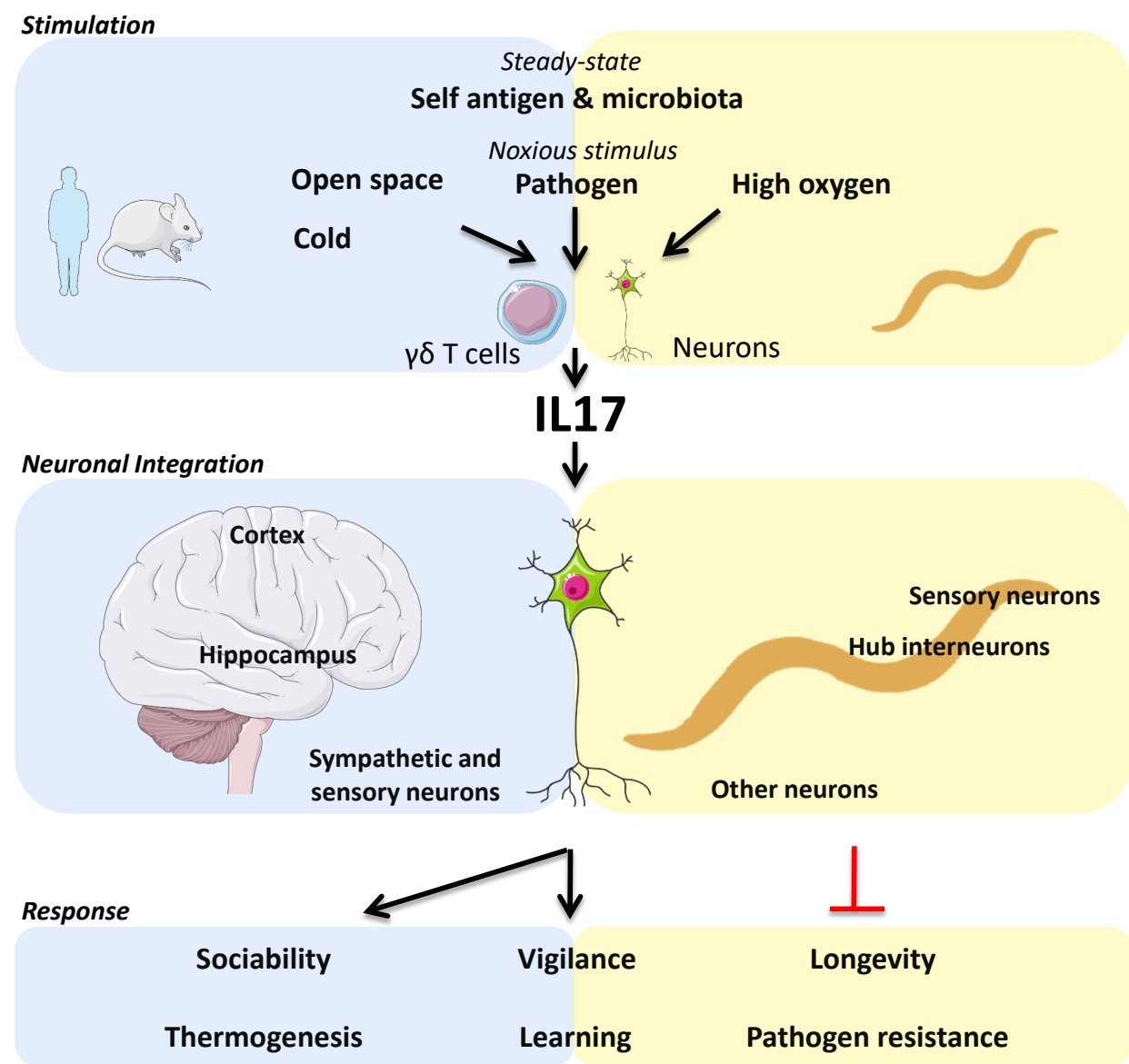

Figure 1. Broad neuronal functions of IL-17s. In mammals (blue), IL-17a is produced at steady state by meningeal $y \delta T$ cells, likely under the influence of self-antigens and the microbiota. IL-17a signalling in the prefrontal cortex prevents risky behaviour in the open-space paradigm, while its action in the hippocampus might promote learning and short-term memory. $\mathrm{IL}-17 \mathrm{a}$ is also induced upon pathogen exposure in the adult, acts on the cortical area and promotes social behaviour in a mouse model of autism. In contrast, similar signalling during the prenatal period decreases social activity. Another member of the IL-17 family, IL-17f, is produced by peripheral $\gamma \delta T$ cells upon cold exposure and promotes sympathetic innervation of adipose tissue, resulting in increased thermogenesis. In the invertebrate $C$. elegans (yellow), IL-17c1, one homolog of IL-17, is produced by neurons at steady state. Upon oxygen exposure, $\mathrm{IL}-17 \mathrm{c} 1$ reinforces the response of hub interneurons to oxygen. This promotes an escape behaviour and avoidance of this noxious stimulus. It also acts on taste-sensing neurons to promote associative learning, such as avoiding chemical cues (salt) linked with a negative outcome (food withdrawal). But in contrast to its protective role in mammalian immunity, neuronal production of IL-17 decreases pathogen resistance and longevity in C. elegans. 